\title{
An Approach to Evaluate the Clothing Creative Design with Dual Hesitant Fuzzy Information
}

\author{
Ya-Mei Li \\ Shandong Management University, Shandong, Jinan 250357, China \\ Correspondence should be addressed to Ya-Mei Li; yamei_li20000@163.com
}

Received 28 June 2014; Accepted 4 July 2014; Published 13 July 2014

Academic Editor: Guiwu Wei

Copyright (C) 2014 Ya-Mei Li. This is an open access article distributed under the Creative Commons Attribution License, which permits unrestricted use, distribution, and reproduction in any medium, provided the original work is properly cited.

\begin{abstract}
The problem of evaluating the clothing creative design with dual hesitant fuzzy information is the multiple attribute decision making problem. In this paper, we have utilized dual hesitant fuzzy hybrid average (DHFHA) operator to develop the model to solve the multiple attribute decision making problems for evaluating the clothing creative design. Finally, a practical example for evaluating the clothing creative design is given to verify the developed approach.
\end{abstract}

\section{Introduction}

In the context of innovation-driven reformation and development of fashion industry in China, it becomes the most essential issue to enhance the ability of independent R\&D and creative design level for Chinese local fashion brands. In quite a long period, fashion is considered to be determined by fashion designers $[1,2]$. However, fashion is hereby considered to be formed according to certain social background, instead of being determined by certain people's subjective minds. So fashion could be generated by precise analysis from objective factors. Now in the context of fast fashion, fashion design does not merely rely on the designers' creativity, but all kinds of modern information technology are applied in the process of fashion design [3-5]. According to the characteristics of the fashion data warehouse system, an overall structure composed of fashion data dictionary, fashion data sources, fashion data management, fashion data mining, and the front-end decision support is formed. The proposed concept of Fashion Data Dictionary (FDD), including Fashion Color Data Dictionary, Fashion Material Data Dictionary, Fashion Accessory Data Dictionary, Fashion Pattern Data Dictionary, Fashion Technique Data Dictionary, Fashion Style Data Dictionary, and Fashion Look Data Dictionary, is formed, in order that all kinds of fashion data from different sources are unified in format. Each data dictionary regulates its data type, level, content, and standard presentation [6, 7]. Sources of fashion data extraction are fashion clothing, social background, and art works. Fashion clothing data sources include fashion shows, fashion market, fashion brand advertisement, target consumer, fashion e-shop, and fashion and fabric exhibition. Social background data sources include politics, economy, environment, science and technology, sports, and lifestyle. Art works data sources include TV drama, art, design, music, performance art, and literature [8]. The fashion data management is defined including fashion data extraction, naming method, conversion rules, and loading standard, so that the fashion data extracted from a variety of sources could be loaded in the fashion warehouse with standardized data format. Social background has an important impact on the formation of fashion style which is the consensus of the fashion industry, but the study of the relationship between the two has always been to stay in the sociology of qualitative research [9-11].

The problem of evaluating the clothing creative design with dual hesitant fuzzy information is the multiple attribute decision making problems. In this paper, we have utilized dual hesitant fuzzy hybrid average (DHFHA) operator to develop the model to solve the multiple attribute decision making problems for evaluating the clothing creative design. Finally, a practical example for evaluating the evaluating the clothing creative design is given to verify the developed approach. 


\section{Preliminaries}

Definition 1 (see [12]). Let $X$ be a fixed set; then a dual hesitant fuzzy set (DHFS) on $X$ is described as

$$
D=(\langle x, h(x), g(x)\rangle \mid x \in X),
$$

in which $h(x)$ and $g(x)$ are two sets of some values in $[0,1]$, denoting the possible membership degrees and nonmembership degrees of the element $x \in X$ to the set $D$, respectively, with the conditions

$$
0 \leq \gamma, \quad \eta \leq 1, \quad 0 \leq \gamma^{+}, \quad \eta^{+} \leq 1,
$$

where $\gamma \in h(x), \eta \in g(x), \gamma^{+} \in h^{+}(x)=\bigcup_{\gamma \in h(x)} \max \{\gamma\}$, and $\eta^{+} \in g^{+}(x)=\bigcup_{\eta \in g(x)} \max \{\eta\}$ for all $x \in X$.

In the following, Wang et al. [13] had developed some dual hesitant fuzzy arithmetic aggregation operator based on the operations of DHFEs.

Definition 2 (see [13]). Let $\widetilde{d}_{j}(j=1,2, \ldots, n)$ be a collection of DHFEs; then their aggregated value by using the DHFWA operator is also a DHFE, and

$$
\begin{aligned}
& \operatorname{DHFWA}_{\omega}\left(\tilde{d}_{1}, \tilde{d}_{2}, \ldots, \tilde{d}_{n}\right) \\
& =\bigoplus_{j=1}^{n}\left(\omega_{j} \tilde{d}_{j}\right) \\
& =\bigcup_{\tilde{\gamma}_{j} \in \tilde{h}_{j}, \eta_{j} \in g_{j}}\left\{\left\{1-\prod_{j=1}^{n}\left(1-\gamma_{j}\right)^{\omega_{j}}\right\},\left\{\prod_{j=1}^{n}\left(\eta_{j}\right)^{\omega_{j}}\right\}\right\},
\end{aligned}
$$

where $\omega=\left(\omega_{1}, \omega_{2}, \ldots, \omega_{n}\right)^{T}$ is the weight vector of $\widetilde{h}_{j}(j=$ $1,2, \ldots, n)$, and $\omega_{j}>0, \sum_{j=1}^{n} \omega_{j}=1$.

Definition 3 (see [13]). Let $\widetilde{d}_{j}(j=1,2, \ldots, n)$ be a collection of DHFEs; then their aggregated value by using the DHFOWA operator is also a DHFE, and

$$
\begin{aligned}
& \operatorname{DHFOWA}_{w}\left(\widetilde{d}_{1}, \widetilde{d}_{2}, \ldots, \widetilde{d}_{n}\right) \\
& =\bigoplus_{j=1}^{n}\left(w_{j} \tilde{d}_{\sigma(j)}\right) \\
& =\bigcup_{\gamma_{\sigma(j)} \in h_{\sigma(j)}, \eta_{\sigma(j)} \in g_{\sigma(j)}}\left\{\left\{1-\prod_{j=1}^{n}\left(1-\gamma_{\sigma(j)}\right)^{w_{j}}\right\},\right. \\
& \left.\left\{\prod_{j=1}^{n}\left(\eta_{\sigma(j)}\right)^{w_{j}}\right\}\right\}
\end{aligned}
$$

where $(\sigma(1), \sigma(2), \ldots, \sigma(n))$ is a permutation of $(1,2, \ldots, n)$, such that $\widetilde{d}_{\sigma(j-1)} \geq \widetilde{d}_{\sigma(j)}$ for all $j=2, \ldots, n$, and $w=$ $\left(w_{1}, w_{2}, \ldots, w_{n}\right)^{T}$ is the aggregation-associated weight vector such that $w_{j} \in[0,1]$ and $\sum_{j=1}^{n} w_{j}=1$.
Definition 4 (see [13]). Let $\widetilde{d}_{j}(j=1,2, \ldots, n)$ be a collection of DHFEs; then their aggregated value by using the DHFHA operator is also a DHFE, and

$$
\begin{aligned}
& \operatorname{DHFHA}_{w, \omega}\left(\tilde{d}_{1}, \tilde{d}_{2}, \ldots, \tilde{d}_{n}\right) \\
& =\bigoplus_{j=1}^{n}\left(w_{j} \dot{\vec{d}}_{\sigma(j)}\right) \\
& =\bigcup_{\dot{\gamma}_{\sigma(j)} \in \dot{h}_{\sigma(j),} \dot{\eta}_{\sigma(j)} \in \dot{g}_{\sigma(j)}}\left\{\left\{1-\prod_{j=1}^{n}\left(1-\dot{\gamma}_{\sigma(j)}\right)^{w_{j}}\right\},\right. \\
& \left.\left\{\prod_{j=1}^{n}\left(\dot{\eta}_{\sigma(j)}\right)^{w_{j}}\right\}\right\}
\end{aligned}
$$

where $w=\left(w_{1}, w_{2}, \ldots, w_{n}\right)$ is the associated weighting vector, with $w_{j} \in[0,1], \sum_{j=1}^{n} w_{j}=1, \dot{h}_{\sigma(j)}$ is the $j$ th largest element of the dual hesitant fuzzy arguments $\dot{\tilde{d}}_{\sigma(j)}\left(\dot{\tilde{d}}_{\sigma(j)}=\right.$ $\left.n \omega_{j} \widetilde{d}_{j}, j=1,2, \ldots, n\right), \omega=\left(\omega_{1}, \omega_{2}, \ldots, \omega_{n}\right)$ is the weighting vector of dual hesitant fuzzy arguments $\tilde{d}_{j}(j=1,2, \ldots, n)$, with $\omega_{i} \in[0,1], \sum_{i=1}^{n} \omega_{i}=1$, and $n$ is the balancing coefficient. In particular, if $w=(1 / n, 1 / n, \ldots, 1 / n)^{T}$, then DHFHA is reduced to the dual hesitant fuzzy weighted average (DHFWA) operator; if $\omega=(1 / n, 1 / n, \ldots, 1 / n)$, then DHFHA is reduced to the dual hesitant fuzzy ordered weighted average (DHFOWA) operator.

Definition 5 (see [12]). Let $d_{i}=\left(h_{d_{i}}, g_{d_{i}}\right)(i=1,2)$ be any two DHFEs, $s(d)=(1 / \# h) \sum_{\gamma \in h} \gamma-(1 / \# g) \sum_{\eta \in g} \eta$ the score function of $d$, and $p(d)=(1 / \# h) \sum_{\gamma \in h} \gamma+(1 / \# g) \sum_{\eta \in g} \eta$ the accuracy function of $d$, where $\# h$ and $\# g$ are the numbers of the elements in $h$ and $g$, respectively.

\section{An Approach to Evaluate the Clothing Creative Design with Dual Hesitant Fuzzy Information}

Let $A=\left\{A_{1}, A_{2}, \ldots, A_{m}\right\}$ be a discrete set of alternatives, and let $G=\left\{G_{1}, G_{2}, \ldots, G_{n}\right\}$ be the state of nature. Suppose that the decision matrix $\widetilde{D}=\left(\widetilde{d}_{i j}\right)_{m \times n}$ is the dual hesitant fuzzy decision matrix, where $\tilde{d}_{i j}(i=1,2, \ldots, m, j=1,2, \ldots, n)$ are in the form of DHFEs.

In the following, we apply the DHFHA operator to the MADM problems for evaluating the clothing creative design with dual hesitant fuzzy information.

Step 1. We utilize the decision information given in matrix $\widetilde{D}$, and the DHFHA operator

$$
\begin{aligned}
\tilde{d}_{i} & =\operatorname{DHFHA}_{w, \omega}\left(\tilde{d}_{i 1}, \tilde{d}_{i 2}, \ldots, \tilde{d}_{i n}\right) \\
& =\bigoplus_{j=1}^{n}\left(w_{j} \dot{\tilde{d}}_{\sigma(i j)}\right)
\end{aligned}
$$


TABLE 1: Dual hesitant fuzzy decision matrix.

\begin{tabular}{ccccc}
\hline & $G_{1}$ & $G_{2}$ & $G_{3}$ & $G_{4}$ \\
\hline$A_{1}$ & $\{\{0.6,0.8\},\{0.4\}\}$ & $\{\{0.3,0.5\},\{0.3\}\}$ & $\{\{0.3\},\{0.6,0.7\}\}$ & $\{\{0.6\},\{0.4\}\}$ \\
$A_{2}$ & $\{\{0.3,0.4\},\{0.5\}\}$ & $\{\{0.3,0.5\},\{0.6\}\}$ & $\{\{0.3,0.4\},\{0.5\}\}$ & $\{\{0.4,0.6\},\{0.4\}\}$ \\
$A_{3}$ & $\{\{0.5,0.7\},\{0.2)\}$ & $\{\{0.4,0.6\},\{0.6\}$ & $\{00.3,0.6\},\{0.2\}\}$ & $\{00.3,0.4\},\{0.5\}\}$ \\
$A_{4}$ & $\{\{0.4,0.5\},\{0.2\}\}$ & $\{\{0.3\},\{0.4,0.5\}\}$ & $\{\{0.3\},\{0.5,0.6\}\}$ & $\{\{0.2\},\{0.3\}\}$ \\
$A_{5}$ & $\{\{0.4\},\{0.4,0.5\}\}$ & $\{\{0.2,0.3\},\{0.5\}\}$ & $\{\{0.3,0.6\},\{0.4\}\}$ & $\{00.4,0.6\},\{0.3\}\}$ \\
\hline
\end{tabular}

$$
\begin{array}{r}
=\bigcup_{\dot{\gamma}_{\sigma(i j)} \in \dot{h}_{\sigma(i)}, \dot{\eta}_{\sigma(i j)} \in \dot{g}_{\sigma(i j)}}\left\{\left\{1-\prod_{j=1}^{n}\left(1-\dot{\gamma}_{\sigma(i j)}\right)^{w_{j}}\right\},\right. \\
\left.\left\{\prod_{j=1}^{n}\left(\dot{\eta}_{\sigma(i j)}\right)^{w_{j}}\right\}\right\}
\end{array}
$$

to derive the overall preference values $\widetilde{d}_{i}(i=1,2, \ldots, m)$ of the alternative $A_{i}$.

Step 2. Calculate the scores $S\left(\widetilde{d}_{i}\right)(i=1,2, \ldots, m)$ of the overall dual hesitant fuzzy preference values $\widetilde{d}_{i}(i=$ $1,2, \ldots, m)$.

Step 3. Rank all the alternatives $A_{i}(i=1,2, \ldots, m)$ and select the best one(s) in accordance with the scores $S\left(\widetilde{d}_{i}\right)(i=$ $1,2, \ldots, m)$.

Step 4. End.

\section{Numerical Example}

Thus, in this section we will present a numerical example for evaluating the clothing creative design with dual hesitant fuzzy information in order to illustrate the method proposed in this paper. There are five possible clothing creative design alternatives $A_{i}(i=1,2,3,4,5)$ for four attributes $G_{j}(j=$ $1,2,3,4)$. The four attributes include the fashion design style $\left(G_{1}\right)$, the color of dress design $\left(G_{2}\right)$, the fabrics of clothing design $\left(G_{3}\right)$, and the design of comfort $\left(G_{4}\right)$, respectively. In order to avoid influencing each other, the decision makers are required to evaluate five possible clothing creative design alternatives $A_{i}(i=1,2,3,4,5)$ under the above four attributes in anonymity and the decision matrix $\widetilde{D}=\left(\widetilde{d}_{i j}\right)_{5 \times 4}$ is presented in Table 1.

In the following, we utilize the approach developed for evaluating the clothing creative design with dual hesitant fuzzy information.

We utilize the decision information given in matrix $\widetilde{D}$ and the DHFHA operator to obtain the overall preference values $\widetilde{d}_{i}$ of the clothing creative design alternatives $A_{i}(i=$ $1,2,3,4,5)$ and calculate the scores $s\left(\tilde{d}_{i}\right)(i=1,2,3,4,5)$ of the overall dual hesitant fuzzy values $\widetilde{d}_{i}(i=1,2,3,4,5)$ of the clothing creative design alternatives $A_{i}$ :

$$
\begin{gathered}
s\left(\widetilde{d}_{1}\right)=0.1324, \quad s\left(\widetilde{d}_{2}\right)=0.1878, \quad s\left(\widetilde{d}_{3}\right)=-0.1022 \\
s\left(\widetilde{d}_{4}\right)=0.3768, \quad s\left(\widetilde{d}_{5}\right)=0.0256 .
\end{gathered}
$$

Then, we rank all the clothing creative design alternatives in accordance with the scores $s\left(\widetilde{d}_{i}\right)(i=1,2,3,4,5)$ of the $\tilde{d}_{i}(i=1,2, \ldots, 5): A_{4}>A_{2}>A_{1}>A_{5}>A_{3}$, and thus the most desirable clothing creative design alternative is $A_{4}$.

\section{Conclusion}

In this paper, we have utilized dual hesitant fuzzy hybrid average (DHFHA) operator to develop the model to solve the multiple attribute decision making problems for evaluating the clothing creative design. Finally, a practical example for evaluating the clothing creative design is given to verify the developed approach.

\section{Conflict of Interests}

The author declares that there is no conflict of interests regarding the publication of this paper.

\section{References}

[1] S. M. Chen, L. W. Lee, H. C. Liu, and S. W. Yang, "Multiattribute decision making based on interval-valued intuitionistic fuzzy values," Expert Systems with Applications, vol. 39, no. 12, pp. 10343-10351, 2012.

[2] T.-Y. Chen, "Nonlinear assignment-based methods for intervalvalued intuitionistic fuzzy multi-criteria decision analysis with incomplete preference information," International Journal of Information Technology \& Decision Making, vol. 11, no. 4, pp. 821-855, 2012.

[3] Z. Zhang, "An interval-valued rough intuitionistic fuzzy set model," International Journal of General Systems, vol. 39, no. 2, pp. 135-164, 2010.

[4] Z. P. Chen and W. Yang, "A new multiple attribute group decision making method in intuitionistic fuzzy setting," Applied Mathematical Modelling, vol. 35, no. 9, pp. 4424-4437, 2011.

[5] X. Zhao, Q. Li, and G. Wei, "Model for multiple attribute decision making on the Einstein correlated information fusion with hesitant fuzzy information," Journal of Intelligent and Fuzzy Systems, vol. 26, no. 6, pp. 3057-3064, 2014. 
[6] D. G. Park, Y. C. Kwun, J. H. Park, and Y. Park II, "Correlation coefficient of interval-valued intuitionistic fuzzy sets and its application to multiple attribute group decision making problems," Mathematical and Computer Modelling, vol. 50, no. 9-10, pp. 1279-1293, 2009.

[7] J. Ye, "Fuzzy decision-making method based on the weighted correlation coefficient under intuitionistic fuzzy environment," European Journal of Operational Research, vol. 205, no. 1, pp. 202-204, 2010.

[8] E. A. Hernandez and V. Uddameri, "Selecting agricultural best management practices for water conservation and quality improvements using atanassov's intuitionistic fuzzy sets," Water Resources Management, vol. 24, no. 15, pp. 4589-4612, 2010.

[9] W. L. Hung and M. S. Yang, "On the J-divergence of intuitionistic fuzzy sets with its application to pattern recognition," Information Sciences, vol. 178, no. 6, pp. 1641-1650, 2008.

[10] Z. S. Xu, "Intuitionistic fuzzy aggregation operators," IEEE Transactions on Fuzzy Systems, vol. 15, no. 6, pp. 1179-1187, 2007.

[11] C. L. Hwang and K. Yoon, Multiple Attribute Decision Making: Methods and Applications, Springer, Berlin, Germany, 1981.

[12] B. Zhu, Z. Xu, and M. Xia, "Dual hesitant fuzzy sets," Journal of Applied Mathematics, vol. 2012, Article ID 879629, 13 pages, 2012.

[13] H. Wang, X. Zhao, and G. Wei, "Dual hesitant fuzzy aggregation operators in multiple attribute decision making," Journal of Intelligent and Fuzzy Systems, vol. 26, no. 5, pp. 2281-2290, 2014. 

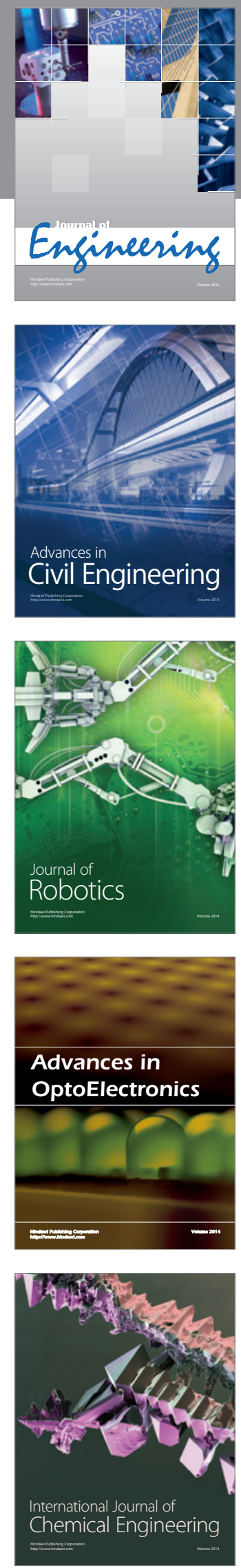

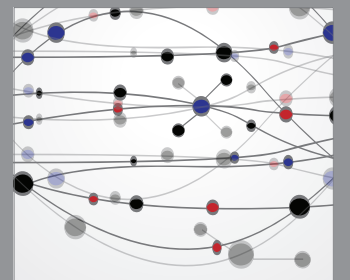

The Scientific World Journal
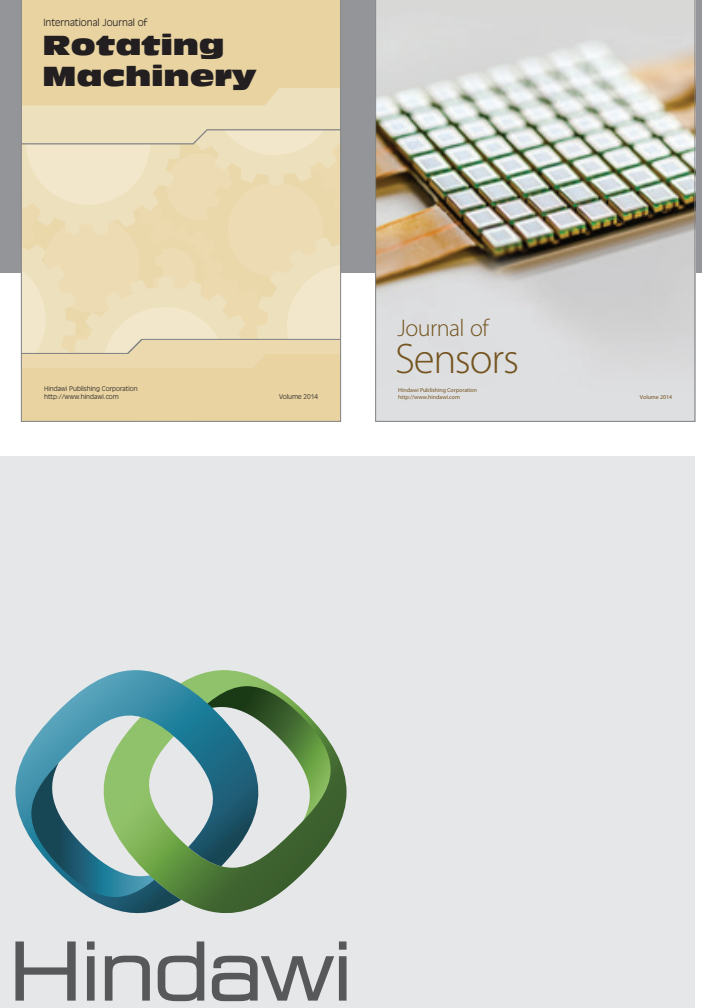

Submit your manuscripts at http://www.hindawi.com
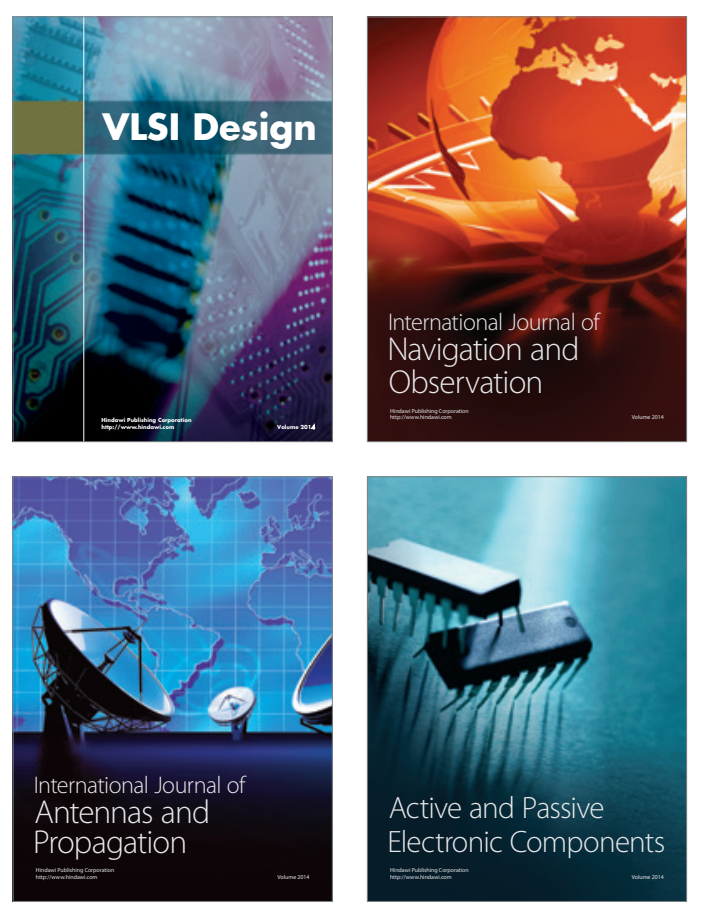
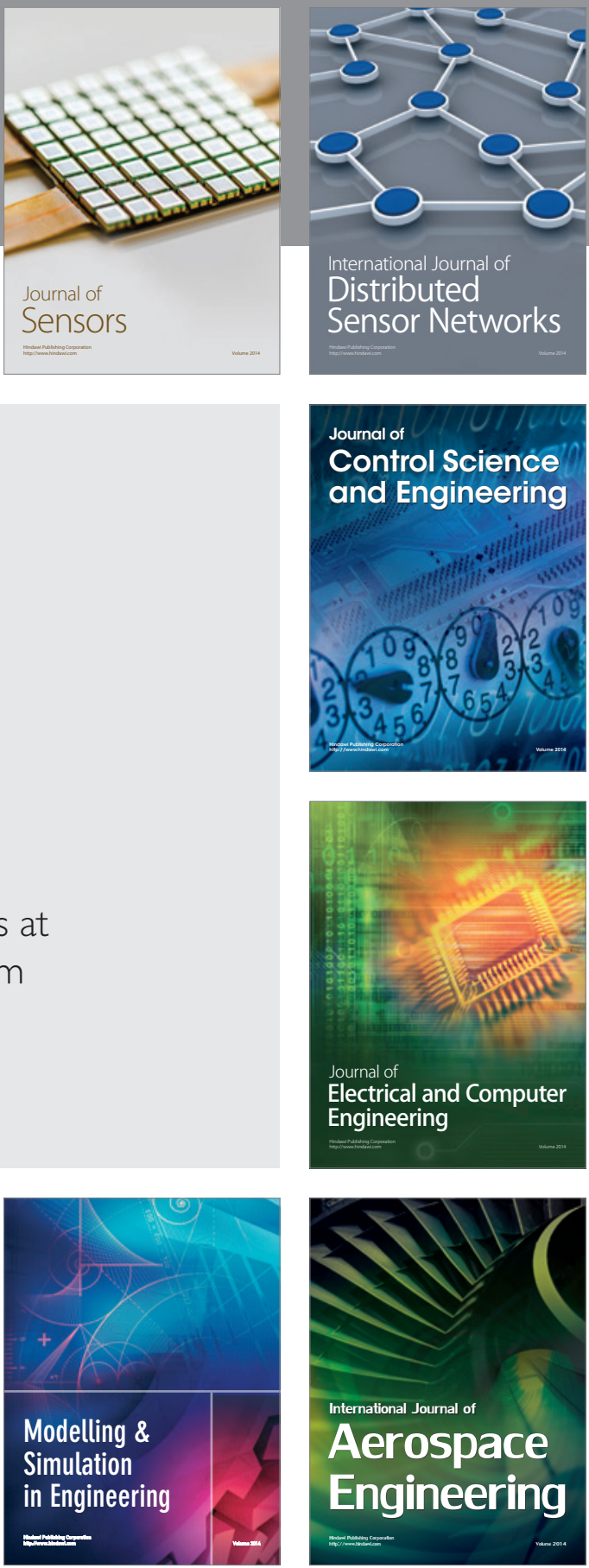

Journal of

Control Science

and Engineering
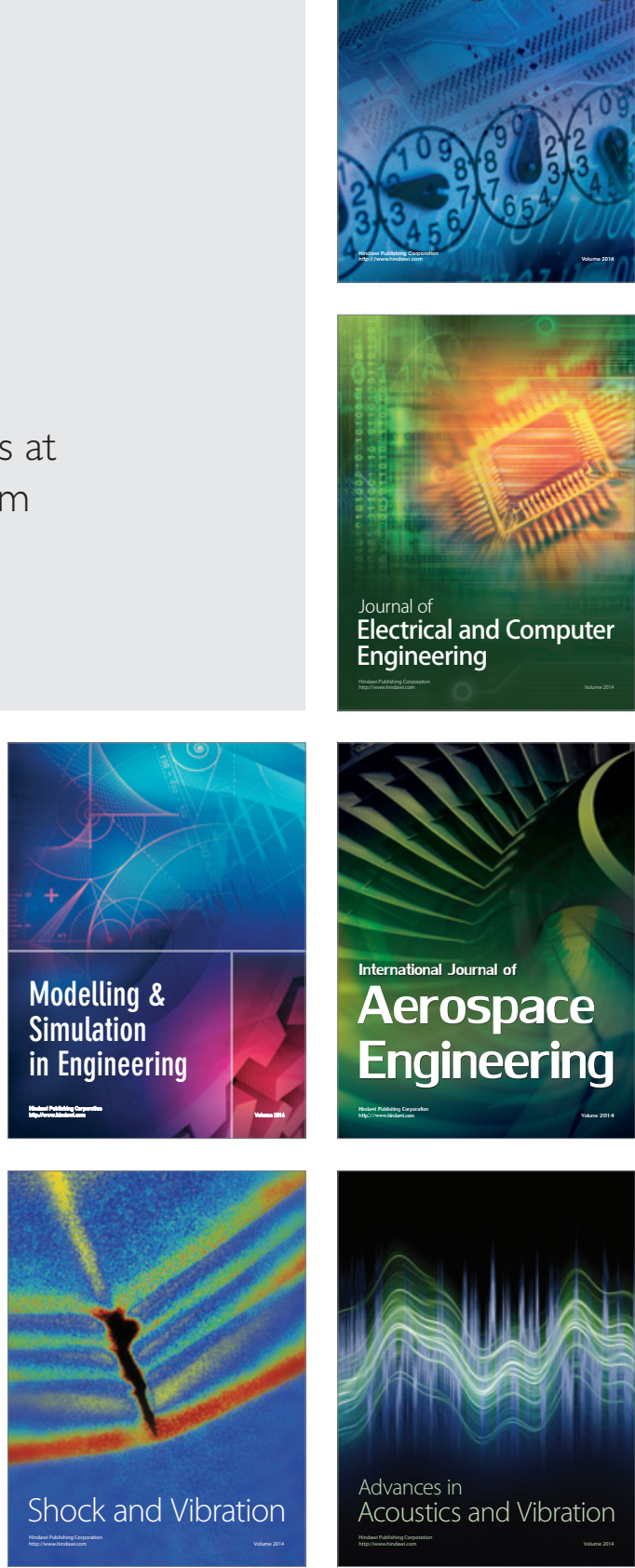